\title{
Metacontrast and paracontrast: Both photopic and scotopic luminance levels yield monotones
}

\author{
LESTER A. LEFTON and YALE NEWMAN \\ University of South Carolina, Columbia, South Carolina 29208
}

\begin{abstract}
Metacontrast and paracontrast were examined under a wide range of luminances and interstimulus intervals. Results show monotonic masking functions for both metacontrast and paracontrast. Luminance affected the detectability of targets and was shown to have small but significant effects on the extent, but not on the nature, of metacontrast and paracontrast functions.
\end{abstract}

Metacontrast has been shown to be sensitive to a large number of variables, with changes in the shape of metacontrast functions attributed to both stimulus and task variables. For example, it has been suggested that the luminance of the stimuli will affect the results obtained (Kolers, 1962) and task variables will also affect results (Lefton, Note 1). Both U-shaped and monotonic metacontrast functions have been obtained under similar stimulus conditions. When a masking annulus is delayed in a metacontrast paradigm, some studies have found U-shaped metacontrast functions (e.g., Weisstein \& Haber, 1965), while others have found monotonic metacontrast functions (e.g., Lefton, 1973).

Metacontrast is often compared to backward masking because the masking stimulus acts backwards on a previously presented target. The difference between metacontrast and backward masking is that metacontrast involves spatially nonoverlapping stimuli, whereas in backward masking the stimuli are spatially overlapping. In a similar manner, paracontrast is often likened to forward masking. In forward masking the masking stimulus precedes the target; in paracontrast the masking annulus also precedes the target but is spatially nonoverlapping. Relatively few studies have been conducted on paracontrast; indeed, most have basically been studies of metacontrast which have also included some paracontrast conditions. Studies presenting conditions of paracontrast have generally found little or no paracontrast, and it has generally been assumed that the effects of paracontrast are, at most, minimal compared with those of metacontrast (e.g., Alpern, 1953; Growney, Weisstein, \& Cox, 1975; Weisstein \& Growney, 1969).

Kolers (1962) has suggested that the shape of a metacontrast function will be determined by contrast ratios that are obtained. He suggests that, when the contrast between a target stimulus and its background is less than the ratio in a mask, the likelihood of monotonic

This research was supported by a grant from the National Eye Council, National Institutes of Health to L. Lefton (1 R01 EY 01201-02). No official endorsement of NEI should be inferred. We thank Jack Griffin for help in data collection and analysis. Requests for reprints should be sent to Lester A. Lefton, Department of Psychology, University of South Carolina, Columbia, South Carolina 29208. functions is greater than when the contrast between the two stimuli is limited. In a similar vein, Weisstein (1968) has argued that, when the ratio of energy in a mask to energy in the target is greater than 10:1, monotonic metacontrast functions will be obtained; when it is less than 10:1, U-shaped functions will be obtained. Metacontrast has often been conceptualized as a phenomenon due to the contrast of the stimulus and its mask; thus, the role of energy variables, particularly luminance, has often been suggested as being potentially important.

The purpose of the present study is to compare metacontrast and paracontrast and the potential role of luminance. No study in the last decade has attempted to compare metacontrast and paracontrast within one study in which the focus was a comparison of the two phenomena. Further, the role of the luminance of the target and the mask has similarly been largely ignored. In the present study both photopic and scotopic luminance levels will be used to assess any potential changes in the magnitude and shape of metacontrast and paracontrast functions.

\section{EXPERIMENT I}

\section{Method}

Subjects. Four undergraduates at the University of South Carolina served as subjects. All subjects were paid volunteers and had normal or corrected vision.

Stimuli and apparatus. A black disk on a white field was used as a target stimulus. The diameter of the target disk was $2.54 \mathrm{~cm}$ and, at a distance of $86 \mathrm{~cm}$, it subtended a visual angle of 1.19 deg. The disk was photoprinted on a semigloss white photo paper to produce high contrast. There was a surround ring which was used as a mask for the target. The inner diameter of the ring corresponded to the diameter of the target, subtending the same degree of visual angle. There was zero intercontour distance. The width of the surrounding mask was $1.2 \mathrm{~cm}$, or .80 , inner to outer border. The stimuli were presented in a Gerbrands fourchannel tachistoscope (Model T-4B).

Procedure and design. Upon introduction to the laboratory, subjects were dark adapted for $15 \mathrm{~min}$ to the low ambient room illumination. During this time they helped the experimenter align the target and mask so that the target would fall just within the center of the masking ring. Before testing began in each session, a set of instructions was read. These instructions were: 'In today's session, as I say 'ready' you will look into the tachistoscope and press the button on the small metal box in front of you. The target will appear before [after] the mask. Your task for each trial will be to answer yes when you are 
sure that you saw the target or no if you did not. You will continue until I say 'stop.' The target will appear in several forms: It may be easily seen; it may be quite difficult to see; it may be somewhere in between; it may not even be presented. Your task remains the same-tell me if you saw the target."

The subjects initiated their own trials by pressing a switch. The following sequence of stimulation was then presented monocularly to the left eye: (metacontrast) fixation, target, interstimulus interval, masking, and then fixation; or (paracontrast) fixation, masking ring, interstimulus interval, target, and then fixation. The target was presented just above a centrally located fixation point. The fixation field was always illuminated, except during the time the target or mask was presented. The luminance of the background of all fields was equated at $50.2 \mathrm{~cd} / \mathrm{m}^{2}$. The dependent variable was the duration at which subjects changed from saying they did not see the target to saying they did see the target. With the ascending method of limits, the duration of the stimulus varied on each trial. When the subjects responded "yes," trials continued until the subject produced three successive yeses.

There were two different conditions, paracontrast and metacontrast. Each subject was tested for three experimental sessions per condition, yielding a total of six experimental sessions. Before testing began, each subject served for two practice sessions which were not included in data analyses. The six experimental sessions were alternated and counterbalanced across subjects. Two subjects began with paracontrast; the other two subjects began with metacontrast. On alternating sessions subjects were presented with either meta- or paracontrast.

Each experimental session consisted of testing one condition (paracontrast or metacontrast) at five different interstimulus intervals (ISIs) using the ascending method of limits. Each ISI was tested six times within a session. The ISIs $(0,25,75,100$, and $500 \mathrm{msec}$ ) were varied between each block of trials. Within a session, the order of ISIs was randomized and counterbalanced. With a total of five ISIs and six replications of each ISI within a session, there were a total of 30 testing blocks per session. In this totally within-subjects design, by the end of the experiment each subject would have contributed 18 measurements for each ISI for each condition. To eliminate potential anticipation effects, using the method of limits each series was started at a different duration, and this was randomized and counterbalanced across subjects. The mask duration was always held constant at $200 \mathrm{msec}$. Subjects were also given strict instructions about fixating and reminded several times within a session about the importance of fixation.

\section{Results and Discussion}

A mean target duration threshold was calculated for each subject for each condition and entered into an analysis of variance (condition by ISI by subjects). The results from Experiment $I$ are in the left-hand panel of Figure 1 and show that there was no overall difference between metacontrast and paracontrast $(\mathrm{F}<1)$; the overall duration threshold for metacontrast was $7.1 \mathrm{msec}$ and for paracontrast was $6.7 \mathrm{msec}$. The effect of introducing an ISI between the target and the mask produced strong effects $[F(4,12)=34.87, p<.001]$. As an ISI was introduced, the target duration threshold decreased dramatically and asymptoted at the $75-\mathrm{msec}$ delay of mask. The interaction of ISI by Condition was marginally significant $[F(4,12)=4.09, p \leqslant .03]$. As the figure shows, metacontrast and paracontrast seem to be similar at most delays of the mask, but at the $0-\mathrm{msec}$ delay there was a difference of over $6 \mathrm{msec}$ in the target duration necessary for the subjects to report the target as being seen; these data points are the major

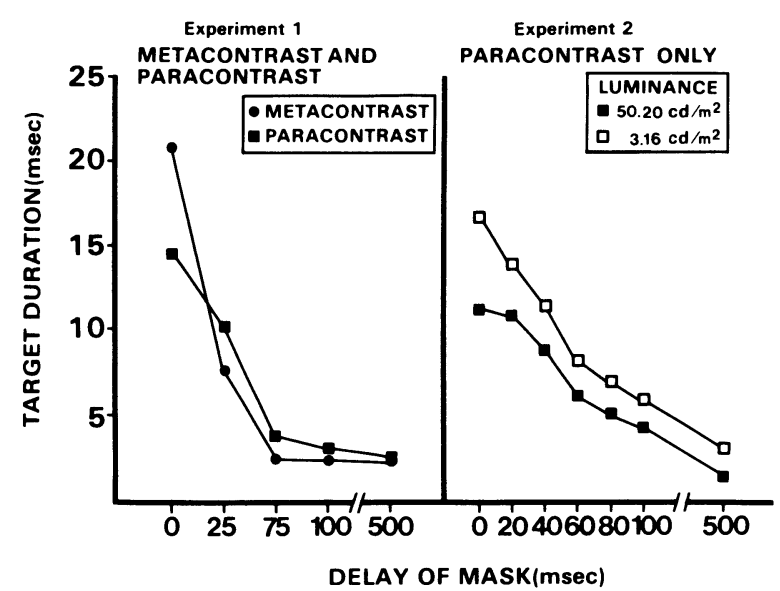

Figure 1. Mean target duration is presented as a function of the delay of the mask for both Experiments I and II. (Each data point in Experiment $I$ represents the mean of 72 observations; in Experiment II each data point represents the mean of 96 observations.)

cause for the interaction. At a 500-msec delay of mask, there was no mask effect in either metacontrast or paracontrast. When a separate analysis was performed on the 500-msec delay of mask to serve as a control condition, this finding was supported because the targets were equally detectable in both conditions $(F<1)$.

The results of Experiment I show that both metaand paracontrast are sensitive to a delay of the mask. While metacontrast seemed to be somewhat stronger than paracontrast at the 0 -msec delay, both meta- and paracontrast were shown to exist and yield monotonic masking functions. Paracontrast is not a weak phenomenon; since paracontrast has generally been considered difficult to obtain, Experiment II was conducted to further explore the existence of paracontrast. In Experiment II paracontrast alone was considered at two different luminance levels.

\section{EXPERIMENT II}

\section{Method}

Subjects. Six undergraduates at the University of South Carolina served as subjects. All were paid volunteers and had normal or corrected vision.

Stimuli, procedure, and design. The same stimuli and procedures as in Experiment I were used. Paracontrast was tested at two luminance levels, $50.2 \mathrm{~cd} / \mathrm{m}^{2}(15 \mathrm{fL})$ and $3.16 \mathrm{~cd} / \mathrm{m}^{2}$ (.95 fL). Each luminance level was tested for one practice and four experimental sessions; therefore, each subject was tested for 10 sessions. Three subjects received the five high-luminancelevel testing sessions first and then the low luminance level; the other three subjects received the opposite order.

Each experimental session consisted of one luminance level tested at seven ISIs. Each ISI was tested four times within each session, with order randomized and counterbalanced. There were two practice blocks at the beginning of each session. Again, the dependent variable was the duration at which the subjects changed from saying they did not see the target to saying they did see the target. The ISIs tested were $0,20,40,60,80,100$, and $500 \mathrm{msec}$.

As in Experiment I, this was a totally within-subjects design. 
Each subject saw both levels of luminance at all seven ISIs four times per session for each of four sessions. Thus, by the end of the experiment each subject would have contributed 16 measurements per ISI.

\section{Results and Discussion}

A mean duration threshold was calculated for each subject for each experimental condition and entered into an analysis of variance (luminance by ISI by subjects). As shown in the right-hand panel of Figure 1, paracontrast again was dramatically evident. As a mask was delayed, there were dramatic effects such that duration threshold decreased $[F(6,20)=5.33, p<.001]$. The effects of luminance were only marginally significant $[F(1,5)=5.02, p \leqslant .074]$. There was an overall difference of about $2.5 \mathrm{msec}$ between the high- and lowluminance paracontrast conditions. This luminance difference was not consistent across all delays of the mask, as evidenced by the small but significant interaction of ISI by luminance $[\mathrm{F}(6,30)=3.00, \mathrm{p}<.05]$. The effect of luminance on paracontrast is probably due to the overall detectability of the target, not to paracontrast itself. When the control condition of the 500 -msec delay of mask was entered into an analysis to see if the target was differentially detectable under the two different luminance levels, a significant effect of luminance was obtained $[F(1,5)=12.62, p<.05]$; the low-luminance target was more difficult to see, whether it was masked or unmasked. While the slopes of the two paracontrast functions are different (luminance by ISI), this is probably due to the difference at the 0 -msec delay of mask.

The purpose of Experiment II was to investigate the viability of paracontrast and to see if and how it responded under photopic and scotopic luminance conditions. As can be seen from the right-hand panel of Figure 1, paracontrast is robust, holds up under photopic and scotopic luminance and generally replicates the results found in Experiment I. The luminance level in Experiment I for paracontrast w'as identical to the high-luminance condition in Experiment II. While the curves with filled-in square symbols do not overlap identically, they clearly show a similar type of function. Paracontrast was again exhibited and it was of about the same strength as in the previous experiment; paracontrast exists and is replicable.

Experiment II examined paracontrast under different conditions of luminance. The purpose of Experiment III was to examine metacontrast under different conditions of luminance. This was done to test the notion that photopic and scotopic luminance conditions might change the shape of metacontrast functions.

\section{EXPERIMENT III}

\section{Method}

Subjects. Six undergraduates at the University of South Carolina served as subjects. All subjects were paid volunteers and had normal or corrected vision.

Stimuli, procedure, and design. The stimuli were the same as those used in Experiments I and II. In this metacontrast para- digm, each subject saw the target and mask at four luminance levels. Neutral-density filters were used to achieve luminance levels of $50.2,12.5,3.16$, and $.25 \mathrm{~cd} / \mathrm{m}^{2}$. Each luminance level was tested for four experimental sessions, so that each subject served for 16 sessions (four luminance levels by four sessions per subject). The luminance levels were randomized and counterbalanced across subjects in order to reduce practice effects.

Each experimental session consisted of the testing of one luminance level at seven ISIs. The ascending method of limits was used to obtain a duration threshold. The order of ISIs within a session was randomized. Each ISI was presented three times within each experimental session. Thus, each ISI for each luminance level yielded 12 observations for each subject.

The subjects initiated their own trials by pressing a switch. The following sequence of stimulation was then presented monocularly to the right eye: fixation, target, ISI, masking ring, and then fixation. The ISIs used were $0,20,40,60,80,100$, and $200 \mathrm{msec}$. The mask was always presented for $200 \mathrm{msec}$. From pilot data a threshold was established on each subject at each ISI. Thus, a starting duration was established for each ISI at each luminance level. With the ascending method of limits, the duration of the stimulus varied on each trial. For 0 - and 20-msec delay, the duration increase between trials was $5 \mathrm{msec}$; for 40 - and 60-msec delay, the increase was $2 \mathrm{msec}$; for 80 -, $100-$, and 200-msec delay, the increase was $1 \mathrm{msec}$. In all other respects, this experiment was identical to Experiments I and II.

\section{Results and Discussion}

As in Experiments I and II, a mean duration threshold was calculated for each subject for each condition and entered into an analysis of variance (luminance by ISI by subjects). All of the main effects and interactions were significant at the .001 level or beyond. Luminance made a considerable difference in metacontrast, such that the low-luminance targets and mask were very difficult to detect and, therefore, showed a considerable amount of metacontrast compared with the higher luminance targets $[F(3,12)=132.98]$. The effect of ISIs was significant; as the mask was delayed, the duration of the target necessary for the subject to say it was present decreased $[F(6,24)=96.51]$. The interaction of luminance by ISI was also significant, although the size of this effect was markedly reduced compared to the main effects $[F(18,72)=3.80]$. As can be seen from Figure 2, when the luminance was clearly photopic lat about $50.2 \mathrm{~cd} / \mathrm{m}^{2}$ or when it was scotopic at $3.16 \mathrm{~cd} / \mathrm{m}^{2}$, the effect of luminance was not large; indeed it resembles the same situation that occurred in the paracontrast study, Experiment II. Yet, when the luminance was reduced another log unit, to $.25 \mathrm{~cd} / \mathrm{m}^{2}$, there was a marked increase in the duration threshold. The highly significant $F$ value of 132 for the luminance effect is largely attributable to this set of data points, which are markedly different from the three higher luminance levels.

As in Experiments I and II monotonic masking functions were obtained. The interaction of luminance by ISI, although significant, was not particularly large; the four metacontrast functions in Figure 2 have relatively similar slope and show that, while luminance affects metacontrast and is differentially affected by the delay of the mask, the shapes of the curves are all still monotones. When the $200-\mathrm{msec}$ control condition 


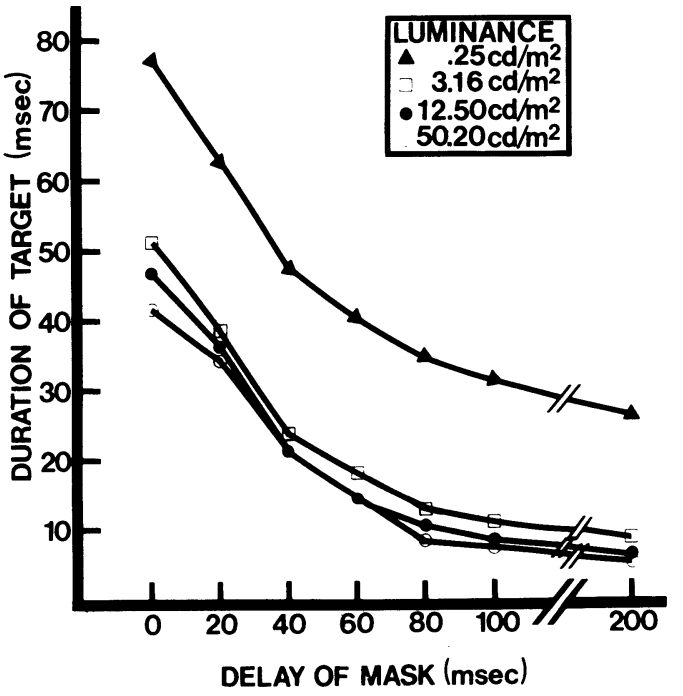

Figure 2. Mean target duration is presented as a function of the delay of the mask for the four luminance levels tested in this metacontrast paradigm, Experiment III. (Each data point represents the mean of 72 observations.)

was entered into a separate analysis, the results showed that the different luminance levels were indeed having their effect on the detectability of the target; the lowluminance-level target was significantly harder to see than the higher-luminance target $[F(2,12)=132.98$, $\mathrm{p}<.001]$. The ordering of the four luminance levels for the $500-\mathrm{msec}$ control condition was appropriate; the highest luminance was most easily detected.

\section{GENERAL DISCUSSION}

The purpose of the present investigation was twofold. First, an attempt was made to examine the strength of paracontrast when compared with metacontrast within a single experimental design. In both Experiments I and II, paracontrast was shown to be strong and replicable. While most studies have shown little or no paracontrast, the present investigation shows that paracontrast is almost as strong as metacontrast. The interaction of a delay of the mask with conditions of meta- and paracontrast show a slightly stronger metacontrast at shorter delays of the mask, but at longer delays the two functions overlap or reverse. Paracontrast exists and it is relatively strong.

The second purpose of the present study was to examine the role of luminance in meta- and paracontrast. Experiment II examined the role of luminance in paracontrast using a clearly photopic and a clearly scotopic luminance. Experiment III examined an even wider range of luminance for metacontrast. In both studies the same effects were evident: The luminance of a target and mask affected detectability; this was clearly shown by the analyses of the control condition, in which there was essentially no masking. The control condition showed that targets of lower luminance were more difficult to detect. When these lower luminance targets were masked through meta- or paracontrast, they were still harder to see. While there were significant differences due to luminance and significant interactions of luminance with ISI, it is clear from the shapes of the functions obtained that luminance affects the overall detectability of the target but it clearly does not change the shape of the functions to any great extent.

It has been suggested that luminance manipulation might change the shape of metacontrast functions. Yet, the present study showed that, regardless of the luminance levels used (either very low scotopic levels or normal photopic levels), monotonic metacontrast and paracontrast functions were obtained.

The shape of meta- and paracontrast functions may be determined by a number of variables. Lefton (Note 1) has suggested that task variables may create the difference. Indeed, he has shown that, when the same stimuli were used but the task was varied, a U-shaped function was obtained for some tasks and monotones for other tasks. It is possible that task parameters and stimulus variables may interact such that highluminance targets may be differentially affected under different tasks. For example, a category estimation task of the clearness of the target may be easier for high-luminance-level stimuli. If this is the case, there could be an interaction of the type of task with luminance.

Luminance affects detectability in both metacontrast and paracontrast, but not enough to worry about the exact luminance levels used in metacontrast studies. Luminance levels in metacontrast studies have varied widely from photopic luminance levels in some studies to scotopic luminance levels in others. The results of the present study show that, at least with a detection task, the luminance level used will not affect the nature of the results obtained to any important degree. Overall duration thresholds will be changed as a function of luminance levels, but few researchers are seriously concerned with the exact performance levels. Generally, they are more concerned with the shape of a function and the relative position of one condition to another. In summary, with detection tasks, both metacontrast and paracontrast seem to be relatively independent of luminance in terms of the shape of the functions obtained.

\section{REFERENCE NOTE}

1. Lefton, L. A. Metacontrast: High-frequency square-wave gratings are difficult to mask. Paper presented at the meetings of the Psychonomic Society, November 7, 1975, Denver, Colorado.

\section{REFERENCES}

AlPERn, M. Metacontrast. Journal of the Optical Society of America, 1953, 43, 648-657.

Growney, R., Weisstein, N., \& Cox, S. I. Measurement of metacontrast. Journal of the Optical Society of America, $1975,65,1379-1381$.

Kolers, P. A. Intensity and contour effects in visual masking. Vision Research, 1962, 2, 277-294.

Lefton, L. A. Spatial factors in metacontrast. Perception \& Psychophysics, 1973, 14, 497-500.

Weisstein, N. A Rashevsky-Landahl neural net: Simulation of metacontrast. Psychological Review, 1968, 75, 494-521.

Weisstein, N., \& Growney, R. L. Apparent movement and metacontrast: A note on Kahneman's formulation. Perception \& Psychophysics, 1969, 5, 321-328.

WeIsSTEIN, N., \& HABER, R. N. A U-shaped backward masking function in vision. Psychonomic Science, 1965, 2, 75-76.

(Received for publication July 30, 1976.) 